\title{
SOME PROPERTIES OF VISCOSITY SOLUTIONS OF HAMILTON-JACOBI EQUATIONS
}

\author{
BY
}

\author{
M. G. CRANDALL ${ }^{1}$, L. C. EVANS ${ }^{2}$ AND P. L. LIONS
}

\begin{abstract}
Recently M. G. Crandall and P. L. Lions introduced the notion of "viscosity solutions" of scalar nonlinear first order partial differential equations. Viscosity solutions need not be differentiable anywhere and thus are not sensitive to the classical problem of the crossing of characteristics. The value of this concept is established by the fact that very general existence, uniqueness and continuous dependence results hold for viscosity solutions of many problems arising in fields of application. The notion of a "viscosity solution" admits several equivalent formulations. Here we look more closely at two of these equivalent criteria and exhibit their virtues by both proving several new facts and reproving various known results in a simpler manner. Moreover, by forsaking technical generality we hereby provide a more congenial introduction to this subject than the original paper.
\end{abstract}

Introduction. Recently two of the authors (see $[2,3]$ ) defined a class of generalized solutions of nonlinear scalar partial differential equations of the form

$$
F(y, u(y), D u(y))=0 \text { for } y \in \mathcal{O},
$$

where $\mathcal{E}$ is an open set in $\mathbf{R}^{m}, F: \Theta \times \mathbf{R} \times \mathbf{R}^{m} \rightarrow \mathbf{R}$ is continuous and $D u=$ $\left(\partial^{u} / \partial_{y_{1}}, \ldots, \partial^{u} / \partial_{y_{m}}\right)$ denotes the gradient of $u$. These generalized solutions-called viscosity solutions in [2] - need not be differentiable anywhere, as the only regularity required in the definition is continuity. M. G. Crandall and P. L. Lions [2] utilized this new concept to establish uniqueness, stability, and certain existence theorems for a wide class of equations of the form (0.1). In addition, P. L. Lions in [8] has extended these techniques to obtain further and more general existence results.

Our goal here is first to look more closely at two alternative definitions of solutions of $(0.1)$, each of which was proved equivalent to the "viscosity" notion in [2], and second to present some new properties of these solutions. Although these alternative definitions were mentioned in [2], they were not used there. Here we emphasize that they are more appealing in some respects and more convenient for certain purposes than the one taken as basic in [2] (see, e.g., Evans [6], which stimulated the current work). In particular, choosing appropriately each time one of

Received by the editors May 27, 1982.

1980 Mathematics Subject Classification. Primary 35F20, 35F25, 35L60.

Key words and phrases. Hamlton-Jacobi equations, uniqueness, generalized solutions.

'Sponsored by the United States Army under Contract No. DAAG-29-80-0041 and supported in part by the National Science Foundation under Grant No. MCS-8002946.

${ }^{2}$ Supported in part by the National Science Foundation under Grant No. MCS-81-02846 and the Alfred P. Sloan Foundation. This work was completed while L. C. Evans was a member of the Institute for Physical Science and Technology, University of Maryland.

(c) 1984 American Mathematical Society $0002-9947 / 84 \$ 1.00+\$ .25$ per page 
these equivalent notions, we can simplify the proofs of several results given in [2]. Furthermore, the concept of viscosity solutions is closely related to some previous work by L. C. Evans [5]. We should also point out that this paper is essentially self-contained and makes easier reading than [2]. as we forsake generality here.

Let us first formulate the definition of viscosity solutions in the form we think the most appealing (even if not always the most convenient to use). We begin hy recalling that a function $u$ from $\theta$ into $\mathbf{R}$ is said to be differentiable at $v_{0} \in \hat{\ominus}$. and that $\operatorname{Du}\left(y_{0}\right)=p_{0} \in \mathbf{R}^{m}$, if we have

$$
u(y)=u\left(y_{0}\right)+p_{0} \cdot\left(y-y_{0}\right)+o\left(\left|y-y_{0}\right|\right) .
$$

Here $a \cdot b$ is the Euclidean scalar product of $a$ and $b$, and $g(y)=o\left(\left|y-y_{0}\right|\right)$ means $\lim _{y \rightarrow y_{0}} g(y)\left|y-y_{0}\right|^{-1}=0$. Obviously, $(0.2)$ is the conjunction of the two relations

$$
\limsup _{y \rightarrow l_{0}}\left(u(y)-u\left(y_{0}\right)-p_{0} \cdot\left(y-y_{0}\right)\right)\left|y-y_{0}\right|^{-1} \leqslant 0
$$

and

$$
\liminf _{y \rightarrow y_{0}}\left(u(y)-u\left(y_{0}\right)-p_{0} \cdot\left(y-y_{0}\right)\right)\left|y-y_{0}\right|^{-1} \geqslant 0 .
$$

It is well known that if $u$ is continuous, it may fail to be differentiable at every $y_{0} \in$ C. Nevertheless, there are-as we will see below-many choices of $\left(y_{0}, p_{0}\right) \in C^{\circ}$ $\times \mathbf{R}^{m}$ for which $(0.3)$ or $(0.4)$ holds. It will thus be convenient to give the following definition.

Definition. Let $u$ be a function from $\varepsilon$ into $\mathbf{R}$ and let $y_{0} \in \hat{\varepsilon}$. Then the superdifferential of $u$ at $y_{0}$ is the set, denoted by $D^{+} u\left(y_{0}\right)$, of $p_{0} \in \mathbf{R}^{m}$ such that (0.3) holds. Similarly, the subdifferential of $u$ at $y_{0}$ is the set, denoted by $D^{-} u\left(v_{0}\right)$. of $p_{0} \in \mathbf{R}^{m}$ such that (0.4) holds.

In general, $D^{ \pm} u\left(y_{0}\right)$ are closed and convex sets. There is an obvious relation between our "subdifferential" and the notion used in convex analysis. We have also learned that the subdifferentials used here were previously employed by $\mathrm{E}$. DeGiorgi, A. Marino and M. Tosques in another context in [4]. We may now define the concept of viscosity solution of $(0.1)$.

DEFINITION 1. A viscosity solution of

$$
F(y, u(y), D u(y))=0 \quad \text { in } 0
$$

is a function $u \in C(\Theta)$ satisfying

$$
F(y, u(y), p) \leqslant 0 \quad \forall y \in\left(, \forall p \in D^{+} u(y),\right.
$$

and

$$
F(y, u(y), p) \geqslant 0 \quad \forall y \in \mathcal{C}, \forall p \in D^{-} u(y) .
$$

In a similar way, $u \in C(\vartheta)$ is said to be a viscosity subsolution (resp. supersolution) of $(0.1)$ if $(0.5)$ (resp. $(0.6))$ holds.

We will reprove in $\$ 1$ that this is equivalent to the following notion of solution of $(0.1)$.

Definition 2. $u \in C(\theta)$ is a viscosity solution of $(0.1)$ provided for all $\phi \in C^{1}(\hat{\imath})$,

$$
\text { if } u-\phi \text { attains a local maximum at } y_{0} \in \Theta \text {, then }
$$

$$
F\left(y_{0}, u\left(y_{0}\right), D \phi\left(y_{0}\right)\right) \leqslant 0
$$


and

$$
\text { if } u-\phi \text { attains a local minimum at } y_{0} \in \mathcal{C} \text {, then }
$$

$$
F\left(y_{0}, u\left(y_{0}\right), D \phi\left(y_{0}\right)\right) \geqslant 0 .
$$

Moreover, each of these notions is equivalent to the one introduced in [2] (see §1).

In $\S 1$ we also establish various properties of viscosity solutions, such as, for example, the consistency with classical solution. $\$ 2$ is devoted to a uniqueness result concerning equations of the type

$$
u+H(D u)=f, \quad x \in \mathbf{R}^{n},
$$

while in $\S 4$ we give a general uniqueness result for the initial value problem

$$
\begin{cases}u_{t}+H(D u)=0, & x \in \mathbf{R}^{n}, t>0, \\ u(x, 0)=u_{0}(x), & x \in \mathbf{R}^{n} .\end{cases}
$$

Here $D u$ denotes the gradient with respect to $x\left(\in \mathbf{R}^{n}\right)$. Of course $(0.10)$ is a special case of $(0.1)$ with $c=\mathbf{R}^{n} \times(0,+\infty), y=(x, t), m=n+1$.

In $\S 3$ we show that the well-known vanishing viscosity method yields viscosity solutions: and finally in $\$ 5$ we consider the relations between viscosity solutions of $(0.10)$ and nonlinear semigroup theory.

Let us recall that many of the results presented here have been already proved in [2], but the proofs herein seems to be simpler (essentially due to our freedom in choosing among the equivalent definitions of viscosity solution). Since the main point here is simplicity, we will not consider more general Hamiltonians $H$ than in $(0.9)$ or (0.10). Technical generality (e.g., $H(x, t, u, D u)$ in $(0.10))$ is available in [2].

\section{Main properties of viscosity solutions.}

\subsection{Equivalent definitions.}

THEOREM 1.1. Let $u \in C(\theta)$. Then the following are equivaleni:

(i) $u$ satisfies $(0.5)$ and $(0.6)$,

(ii) $u$ satisfies (0.7) and (0.8) for all $\phi \in C^{1}(\theta)$,

(iii) $u$ satisfies, for all $\phi \in C_{c}^{1}(\Theta),{ }^{3} \phi \geqslant 0$ and $k \in \mathbf{R}$,

$$
\left\{\begin{array}{l}
\text { if } \max _{\hat{0}} \phi(u-k)>0 \text {, then for some point } y_{0} \text { at which } \phi(u-k) \\
\text { attains its maximum, we have } F\left(y_{0}, u\left(y_{0}\right),-\frac{D \phi\left(y_{0}\right)}{\phi\left(y_{0}\right)}\left(u\left(y_{0}\right)-k\right)\right) \leqslant 0
\end{array}\right.
$$

and

$$
\left\{\begin{array}{l}
\text { if } \min _{\hat{c}} \phi(u-k)<0, \text { then for some point } y_{0} \text { at which } \phi(u-k) \\
\text { attains its minimum, we have } F\left(y_{0}, u\left(y_{0}\right),-\frac{D \phi\left(y_{0}\right)}{\phi\left(y_{0}\right)}\left(u\left(y_{0}\right)-k\right)\right) \geqslant 0 .
\end{array}\right.
$$

\footnotetext{
${ }^{3} C_{C}^{1}(\mathbb{E})=\left\{\phi \in C^{1}(\mathbb{E})\right.$, the support of $\phi$ is a compact subset of $\left.\mathbb{E}\right\}$.
} 
REMARK 1.1. It is easy to prove that in (ii) or (iii) we may replace $\phi \in C^{1}(\mathbb{0})$ by $\phi \in C_{c}^{\infty}(\theta)$. (See the proof of Theorem 3.1.)

REMARK 1.2. Property (iii) is the original definition of viscosity solutions given in [2] (where the equivalence of (i), (ii) and (iii) is also proved).

REMARK 1.3. A more precise statement than Theorem 1.1 asserts the equivalence of: (i) $u$ satisfies (0.5), (ii) $u$ satisfies (0.7) and (iii) $u$ satisfies (1.1). When $u$ satisfies one of these equivalent conditions, it is called a viscosity subsolution of $(0,1)$. An analogous statement holds for supersolutions.

REMARK 1.4. Let us finally mention that we could define the notion of viscosity subsolutions for upper semicontinuous functions and most of the results of [2] would still remain valid.

We will prove (i) $\Leftrightarrow$ (ii). For the equivalence with (iii), see [2]. That (i) $\Leftrightarrow$ (ii) is an immediate consequence of the following

Proposition 1.1. Let $u \in C(\vartheta), y_{0} \in \mathcal{\theta}, p \in \mathbf{R}^{m}$ : Then the following are equivalent:

(i) $p \in D^{+} u\left(y_{0}\right)$ (resp. $p \in D^{-} u\left(y_{0}\right)$ ) and

(ii) there exists $\phi \in C^{1}(\theta)$ such that $u-\phi$ has a local maximum (resp. minimum) at $y_{0}$ and $D \phi\left(y_{0}\right)=p$.

Proof. The key fact is the following form [2, Lemma I.4] of a result of [5]:

Lemma 1.1. Let $\eta \in C(\theta)$ be differentiable at $y_{0} \in \mathcal{\theta}$. Then there exists $\Psi_{ \pm} \in C^{1}(\mathcal{\theta})$ such that $D \Psi_{+}\left(y_{0}\right)=D \Psi_{-}\left(y_{0}\right)=D \eta\left(y_{0}\right)$ and $\eta-\Psi_{+}\left(\eta-\Psi_{-}\right)$has a strict local maximum (resp. minimum) value of zero at $y_{0}$.

Accepting this lemma, we prove Proposition 1.1. Assume $p \in D^{+} u\left(y_{0}\right)$. Set $\eta(y)=\left\{u(y)-u\left(y_{0}\right)-p \cdot\left(y-y_{0}\right)\right\}^{+}$, where $r^{+}$denotes $\max (r, 0)$. By assumption, $\eta$ is differentiable at $y_{0}$ and $D \eta\left(y_{0}\right)=0$. Let $\Psi_{+} \in C^{1}(\theta)$ be as in Lemma 1.1. Then near $y_{0}$,

$$
\left\{u(y)-\left[u\left(y_{0}\right)+p \cdot\left(y-y_{0}\right)\right]\right\}^{+}-\Psi_{+}(y) \leqslant 0 ;
$$

so if $\phi(y)=u\left(y_{0}\right)+p \cdot\left(y-y_{0}\right)+\Psi_{+}(y), u-\phi$ has a local maximum at $y_{0}$ and $D \phi\left(y_{0}\right)=p$. Thus (i) implies (ii). It is clear that (ii) implies (i), since if $u-\phi$ has a maximum at $y_{0}$, then near $y_{0}$ we have

$$
u(y) \leqslant u\left(y_{0}\right)-\phi\left(y_{0}\right)+\phi(y) \leqslant u\left(y_{0}\right)+D \phi\left(y_{0}\right) \cdot\left(y-y_{0}\right)+o\left(\left|y-y_{0}\right|\right) .
$$

This implies $D \phi\left(y_{0}\right) \in D^{+} u\left(y_{0}\right)$. (We have dealt with $D^{+}$and local maxima; the situation for $D^{-}$and local minima is entirely the same.)

1.2. Elementary properties of viscosity solutions. We begin with a simple result which establishes the consistency of the notions of viscosity and classical solutions.

THEOREM 1.2. (i) Let $u \in C^{1}(\theta)$ be a classical solution of $(0.1)$, that is

$$
F(y, u(y), D u(y))=0 \quad \text { in } \theta, u \in C^{1}(\theta) .
$$

Then $u$ is a viscosity solution.

(ii) Let $u$ be a viscosity solution of $(0.1)$ which is differentiable at some $y_{0} \in \mathcal{O}$. Then

$$
F\left(y_{0}, u\left(y_{0}\right), D u\left(y_{0}\right)\right)=0 \text {. }
$$


Remark 1.5. This result is proved in [2, Corollary I.6]. (See also [5, pp. 237-238].) Analogous statements hold for viscosity sub- and supersolutions. Obviously, we deduce from (ii) above that if $u$ is a viscosity solution of $(0.1)$ and if $u \in W_{\mathrm{loc}}^{1, p}(\theta)$ for $p>m$, then

$$
F(y, u, D u)=0 \quad \text { a.e. in } \theta \text {. }
$$

The proof of Theorem 1.2 is trivial, since if $u$ is $C^{1}$, then at every point $y \in \theta$, the super- and the subdifferentials coincide and $D^{+} u(y)=D^{-} u(y)=\{D u(y)\}$. In the same way, if $u$ is a viscosity solution of $(0.1)$ and is differentiable at $y \in \theta$, then $D^{+} u\left(y_{0}\right)=D^{-} u\left(y_{0}\right)=\left\{D u\left(y_{0}\right)\right\}$.

We next reprove Theorem I.10 of [2], which characterizes piecewise $C^{1}$ viscosity solutions of $F=0$. Let $\theta$ be divided in two open subsets $\theta_{+}$and $\theta_{-}$by a $C^{1}$ surface $\Gamma: \hat{\theta}=\theta_{+} \cup \hat{\theta}_{-} \cup \Gamma$. The unit normal to $\Gamma$ at $y_{0} \in \Gamma$ is denoted by $n\left(y_{0}\right)$ and is taken to point into $e^{+}$.

THEOREM 1.3. Let $u \in C(\theta)$ and $u=u_{+}$in $\theta_{+} \cup \Gamma, u=u_{-}$in $\theta_{-} \cup \Gamma$ where $u_{+}, u_{-}$ are of class $C^{1}$ in $\theta_{+} \cup \Gamma$, and $\theta_{-} \cup \Gamma$. Then $u$ is a viscosity solution of $(0.1)$ if and only if the following conditions hold:

(a) $u_{+}$and $u_{-}$are classical solutions of $F=0$ in $\theta_{+}$and $\theta_{-}$respectively, and

(b) if $y_{0} \in \Gamma, T y_{0}=\left\{\tau \in \mathbf{R}^{m}: n\left(y_{0}\right) \cdot \tau=0\right\}$ is the tangent space to $\Gamma$ at $y_{0}$ and $P_{T}$ is the orthogonal projection of $\mathbf{R}^{m}$ onto $T y_{0}$, then

$$
\left\{\begin{array}{l}
\text { if } a=D u_{+}\left(y_{0}\right) \cdot n\left(y_{0}\right) \leqslant D u_{-}\left(y_{0}\right) \cdot n\left(y_{0}\right)=b, \text { we have } \\
F\left(y_{0}, u\left(y_{0}\right), P_{T} D u_{ \pm}\left(y_{0}\right)+\xi n\left(y_{0}\right)\right) \leqslant 0 \text { for } a \leqslant \xi \leqslant b
\end{array}\right.
$$

and

$$
\left\{\begin{array}{l}
\text { if } a=D u_{+}\left(y_{0}\right) \cdot n\left(y_{0}\right) \geqslant D u_{-}\left(y_{0}\right) \cdot n\left(y_{0}\right)=b, \text { we have } \\
F\left(y_{0}, u\left(y_{0}\right), P_{T} D u_{ \pm}\left(y_{0}\right)+\xi n\left(y_{0}\right)\right) \geqslant 0 \text { for } b \leqslant \xi \leqslant a
\end{array}\right.
$$

Proof. First note that the assumptions imply that $P_{T} D u_{+}\left(y_{0}\right)=P_{T} D u_{-}\left(y_{0}\right)$ for $y_{0} \in \Gamma$; hence $P_{T} D u_{ \pm}\left(y_{0}\right)$ is unambiguous. Now, by assumption, if $y \in \theta_{+} \cup \theta_{-}$, then $D^{+} u(y)=D^{-} u(y)=\{D u(y)\}$; therefore (a) is equivalent to the satisfaction of $(0.5)$ and $(0.6)$ for $y \in \mathcal{O} \backslash \Gamma$.

We are now going to prove that (b) is equivalent to (0.5) and (0.6) for $y \in \Gamma$. In order to do so, we just need to compute $D^{+} u\left(y_{0}\right), D^{-} u\left(y_{0}\right)$ for $y_{0} \in \Gamma$. By assumption we have

$$
\begin{aligned}
u(y)= & u\left(y_{0}\right)+P_{T} D u_{+}\left(y_{0}\right) \cdot\left(y-y_{0}\right)+\left(n\left(y_{0}\right) \cdot D u_{+}\left(y_{0}\right)\right) \\
& \cdot\left(n\left(y_{0}\right) \cdot\left(y-y_{0}\right)\right)+o\left(\left|y-y_{0}\right|\right) \quad \text { if } y \in \vartheta_{+} \cup \Gamma
\end{aligned}
$$

and

$$
\begin{aligned}
u(y)= & u\left(y_{0}\right)+P_{T} D u_{-}\left(y_{0}\right) \cdot\left(y-y_{0}\right)+\left(n\left(y_{0}\right) \cdot D u_{-}\left(y_{0}\right)\right) \\
& \cdot\left(n\left(y_{0}\right) \cdot\left(y-y_{0}\right)\right)+o\left(\left|y-y_{0}\right|\right) \quad \text { if } y \in \theta_{-} \cup \Gamma .
\end{aligned}
$$


On the other hand, $p \in D^{+} u\left(y_{0}\right)$ if and only if

$$
\begin{aligned}
u(y) & \leqslant u\left(y_{0}\right)+p \cdot\left(y-y_{0}\right)+o\left(\left|y-y_{0}\right|\right) \\
& =u\left(y_{0}\right)+P_{T} p \cdot\left(y-y_{0}\right)+\left(n\left(y_{0}\right) \cdot p\right)\left(n\left(y_{0}\right) \cdot\left(y-y_{0}\right)\right)+o\left(\left|y-y_{0}\right|\right) .
\end{aligned}
$$

Recalling that $P_{T} D u_{+}\left(y_{0}\right)=P_{T} D u_{-(}\left(y_{0}\right)$ and that $\Gamma$ is of class $C^{\prime}$, one deduces immediately that $p \in D^{+} u\left(y_{0}\right)$ : : and only if

$$
P_{T} p=P_{T} D u .\left(y_{0}\right) \text { and } n\left(y_{0}\right) \cdot D u_{+}\left(y_{0}\right) \leqslant n\left(y_{0}\right) \cdot p \leqslant n\left(y_{0}\right) \cdot D u_{-}\left(y_{0}\right) \text {. }
$$

Thus

$$
D^{+} u\left(y_{0}\right)=\left\{P_{T} D u_{ \pm}\left(y_{0}\right)+\xi n\left(y_{0}\right): n\left(y_{0}\right) \cdot D u_{+}\left(y_{0}\right) \leqslant \xi \leqslant n\left(y_{0}\right) \cdot D u_{--}\left(y_{0}\right)\right\}
$$

(and if $n\left(y_{0}\right) \cdot D u_{+}\left(y_{0}\right)>n\left(y_{0}\right) \cdot D u_{-}\left(y_{0}\right), D^{+} u\left(y_{0}\right)$ is empty). In the same way, we find

$$
D^{-} u\left(y_{0}\right)=\left\{P_{T} D u_{+}\left(y_{0}\right)+\xi n\left(y_{0}\right): n\left(y_{0}\right) \cdot D u_{-}\left(y_{0}\right) \leqslant \xi \leqslant n\left(y_{0}\right) \cdot D u_{+}\left(y_{0}\right)\right\} .
$$

It is then straightforward to complete the proof of Theorem 1.3.

REMARK 1.6. It is worth noting that due to the use of Definition 1 of viscosity solutions, the above proof of Theorem 1.3 is more direct than in [2]. This phenomenon is further illustrated by two easy results (the first in Corollary I.8 and the second in Theorem I.2 of [2]).

Proposition 1.2. Let $u$ be a viscosity solution of $(0.1)$ and let $\Phi \in C^{1}(\mathbf{R})$ satisfy $\Phi^{\prime}(t)>0$ in $\mathbf{R}, \Phi(\mathbf{R})=\mathbf{R}$. Then $\Phi(u)$ is a viscosity solution of

$$
F\left(x, \Psi(v), \Psi^{\prime}(v) D v\right)=0,
$$

where $\Psi$ denotes $\Phi^{-1}$.

Proof. This is immediate from the observations

$$
D^{+} \boldsymbol{\Phi}(u)\left(y_{0}\right)=\Phi^{\prime}\left(u\left(y_{0}\right)\right) D^{+} u\left(y_{0}\right), \quad D^{-} \Phi(u)\left(y_{0}\right)=\Phi^{\prime}\left(u\left(y_{0}\right)\right) D^{-} u\left(y_{0}\right) \text {. }
$$

Proposition 1.3. (a) Let $u, v$ be viscosity subsolutions (resp. supersolutions) of (0.1). Then $w=\max (u, v)($ resp. $w=\min (u, v))$ is a viscosity subsolution (resp. supersolution ) of (0.1).

(b) Let $\left(u_{n}\right)_{n \geqslant 1}$ be viscosity subsolutions (resp. supersolutions) of (0.1). If $w=$ $\sup _{n \geqslant 1} u_{n}$ (resp., $\left.\inf _{n \geqslant 1} u_{n}\right) \in C(\vartheta)$, then $w$ is a viscosity subsolution (resp. supersolution) of $(0.1)$.

Proof. The proof of (a) is an immediate consequence of the remark

$$
D^{+}\{\max (u, v)\}\left(y_{0}\right) \subset D^{+} u\left(y_{0}\right) \cup D^{+} v\left(y_{0}\right)
$$

(resp. $\left.D^{-}\{\min (u, v)\}\left(y_{0}\right) \subset D^{-} u\left(y_{0}\right) \cup D^{-} v\left(y_{0}\right)\right)$. And (b) is a consequence of (a) and of the following stability result. Observe that $w_{m}=\sup _{m \geqslant n \geqslant 1} u_{n}$ converges uniformly on compact sets of $\theta$ to $w$, because of Dini's lemma.

THEOREM 1.4. Let $F_{n}(y, t, p)$ be a sequence of continuous functions such that $F_{n}(y, t, p)$ converges uniformly on compact subsets of $\Theta \times \mathbf{R} \times \mathbf{R}^{m}$ to some function 
$F(v, t, p)$. Let $u_{n}$ be a viscosity solution of $F_{n}\left(y, u_{n}, D u_{n}\right)=0$ in E. We assume that $u_{n}$ converges uniformly on compact subsets of $\mathbb{E}$ to some $u$. Then $u$ is a viscosity solution of $F(y, u, D u)=0$.

Pruuf. By Theorem 1.1, we need only to consider points of local maximum of $u-\phi$ for $\phi \in C^{\prime}(\varepsilon)$. Let $y_{0}$ be such a point and let us prove that

$$
F\left(y_{0}, u\left(y_{0}\right), D \phi\left(y_{0}\right)\right) \leqslant 0 \text {. }
$$

Take $\Psi \in C^{\prime \prime}\left(C^{\prime}\right)$ such that $0 \leqslant \Psi<1$ if $y \neq y_{0}$ and $\Psi\left(y_{0}\right)=1$. It is clear that $u-(\phi-\Psi)$ attains a local strict maximum at $y_{0}$ and thus for $n$ large enough, there will exist $y_{n}$ such that $u_{n}-(\phi-\Psi)$ attains a local maximum at $y_{n}, y_{n} \in \mathcal{\theta}$, and $y_{n} \rightarrow y_{0}$. By assumption, we have

$$
F_{n}\left(y_{n}, u_{n}\left(y_{n}\right), D \phi\left(y_{n}\right)-D \Psi\left(y_{n}\right)\right) \leqslant 0,
$$

and we conclude since $u_{n}\left(y_{n}\right) \rightarrow u\left(y_{0}\right)$ and $D \phi\left(y_{n}\right)-D \Psi\left(y_{n}\right) \rightarrow D \phi\left(y_{0}\right)-D \Psi\left(y_{0}\right)$ $=D \phi\left(y_{0}\right)$.

REMARK 1.7. The proof above actually shows that if $u_{n} \rightarrow u$ in $C\left(\mathbb{C}^{\circ}\right)$, then for all $p \in D^{+} u\left(y_{0}\right)$ (resp. $\left.D u\left(y_{0}\right)\right)$ there exist $y_{n} \in \mathcal{E}, p_{n} \in D^{+} u_{n}\left(y_{n}\right)$ (resp $\left.D^{-} u_{n}\left(y_{n}\right)\right)$ such that $y_{n} \rightarrow y_{0}, p_{n} \rightarrow p$. In other words,

$$
D^{+} u\left(y_{0}\right) \subset \limsup _{\substack{n \rightarrow \infty \\ y \rightarrow y_{0}}} D^{+} u_{n}(y) \text {. }
$$

2. Uniqueness for $u+H(D u)=f$ in $\mathbf{R}^{n}$. We now consider the uniqueness of viscosity solutions of

$$
u+H(D u)=f \text { in } \mathbf{R}^{n} .
$$

Of course, (2.1) is a very special case of $(0.1)$ : take $m=n, y=x, F(y, r, p)=r+$ $H(p)-f(y)$. We will prove the following

Theorem 2.1. Let $u, v, f, g, H \in C\left(\mathbf{R}^{n}\right)$. Assume that $u$, $v$ are bounded and $f, g$ are uniformly continuous on $\mathbf{R}^{n}$. Assume that $u$ and $v$ are viscosity solutions of, respectively, $u+H(D u)=f, v+H(D v)=g$ in $\mathbf{R}^{n}$. Then we have

$$
\sup _{\mathbf{R}^{n}}(u-v)^{+} \leqslant \sup _{\mathbf{R}^{n}}(f-g)^{+} .
$$

REMARK 2.1. By symmetry, we also have $\sup _{\mathbf{R}^{\prime \prime}}(v-u)^{+} \leqslant \sup _{\mathbf{R}^{n}}(g-f)^{+}$and thus $\|u-v\| \leqslant\|f-g\|$, where $\|h\|=\sup _{\mathbf{R}^{n}}|h(x)|$ is the norm on the space $C_{b}\left(\mathbf{R}^{n}\right)$ of bounded continuous functions on $\mathbf{R}^{n}$.

Proof of THEOREM 2.1. We begin the proof by assuming the extra conditions

$$
\lim _{|x| \rightarrow \infty} u(x)=\lim _{|x| \rightarrow \infty} v(x)=0 .
$$

This will keep the ideas clearer; later the full result is established.

We choose a function $\beta \in C^{\infty}\left(\mathbf{R}^{n}\right)$ with the properties

$$
0 \leqslant \beta \leqslant 1, \quad \beta(0)=1, \quad \beta(x)=0 \quad \text { if }|x|>1 .
$$

Let $M=\max (\|u\|,\|v\|), \varepsilon>0$, and let $\Phi: \mathbf{R}^{n} \times \mathbf{R}^{n} \rightarrow \mathbf{R}$ be given by

$$
\Phi(x, y)=u(x)-v(y)+3 M \beta_{\varepsilon}(x-y),
$$


where

$$
\beta_{\varepsilon}(z)=\beta(z / \varepsilon) \text { for } z \in \mathbf{R}^{n} .
$$

By (2.3)-(2.6) and the choice of $\beta_{\varepsilon}, \lim \sup _{|x|+|y| \rightarrow \infty} \Phi(x, y) \leqslant 3 M$. (In fact, off the support of $\beta_{\varepsilon}(x-y), \Phi \leqslant 2 M$, while if $|x|+|y| \rightarrow \infty$ on this support, then $|x|$ and $|y| \rightarrow \infty$.) We may assume that $u(\bar{x})>v(\bar{x})$ for some $\bar{x}$, since (2.2) clearly holds if this is not the case. But then

$$
\Phi(\bar{x}, \bar{x})=u(\bar{x})-v(\bar{x})+3 M \beta_{\varepsilon}(0)>3 M .
$$

Therefore $\Phi$ assumes its maximum value at some point $\left(x_{0}, y_{0}\right) \in \mathbf{R}^{n} \times \mathbf{R}^{n}$; i.e.

$$
\begin{aligned}
\Phi\left(x_{0}, y_{0}\right) & =u\left(x_{0}\right)-v\left(y_{0}\right)+3 M \beta_{\varepsilon}\left(x_{0}-y_{0}\right) \\
& \geqslant u(x)-v(y)+3 M \beta_{\varepsilon}(x-y) \text { for all }(x, y) \in \mathbf{R}^{n} \times \mathbf{R}^{n} .
\end{aligned}
$$

Moreover, $\left|x_{0}-y_{0}\right| \leqslant \varepsilon$ since $\beta_{\varepsilon}\left(x_{0}-y_{0}\right)>0$ by the above.

Now $x_{0}$ is a maximum point of $x \mapsto u(x)-\left(v\left(y_{0}\right)-3 M \beta_{\varepsilon}(x-y)\right)$ and thus, by assumption,

$$
u\left(x_{0}\right)+H\left(-3 M\left(D \beta_{\varepsilon}\right)\left(x_{0}-y_{0}\right)\right) \leqslant f\left(x_{0}\right) .
$$

Similarly, $y_{0}$ is a minimum point of $y \mapsto v(y)-\left(u\left(x_{0}\right)+3 M \beta_{\varepsilon}\left(x_{0}-y\right)\right)$ and so

$$
v\left(y_{0}\right)+H\left(-3 M\left(D \beta_{\varepsilon}\right)\left(x_{0}-y_{0}\right)\right) \geqslant g\left(y_{0}\right) \text {. }
$$

Together, (2.8) and (2.9) yield

$$
u\left(x_{0}\right)-v\left(y_{0}\right) \leqslant f\left(x_{0}\right)-g\left(y_{0}\right) .
$$

For $x \in \mathbf{R}^{n}$,

$$
u(x)-v(x)+3 M=\Phi(x, x) \leqslant \Phi\left(x_{0}, y_{0}\right) \leqslant u\left(x_{0}\right)-v\left(y_{0}\right)+3 M
$$

so, by (2.10),

$$
\begin{aligned}
\sup _{\mathbf{R}^{n}}(u(x)-v(x))^{+} & \leqslant\left(u\left(x_{0}\right)-v\left(y_{0}\right)\right)^{+} \leqslant\left(f\left(x_{0}\right)-g\left(y_{0}\right)\right)^{+} \\
& \leqslant \sup _{\mathbf{R}^{n}}(f-g)^{+}+\left|g\left(x_{0}\right)-g\left(y_{0}\right)\right| \\
& \leqslant \sup _{\mathbf{R}^{n}}(f-g)^{+}+\omega_{g}(\varepsilon),
\end{aligned}
$$

where $\omega_{g}(\cdot)$ is the modulus of continuity of $g$. Now (2.2) follows upon our letting $\varepsilon \rightarrow 0$.

The extra assumption (2.3) was used to guarantee that $\Phi$ had a global maximum point $\left(x_{0}, y_{0}\right)$. To treat the general case, choose $\delta>0$ and then $\left(x_{1}, y_{1}\right)$ so that

$$
\Phi\left(x_{1}, y_{1}\right) \geqslant \sup _{\mathbf{R}^{n} \times \mathbf{R}^{n}} \Phi(x, y)-\delta .
$$

Now choose $\zeta \in C^{\infty}\left(\mathbf{R}^{n} \times \mathbf{R}^{n}\right)$ such that

$$
\left\{\begin{array}{l}
0 \leqslant \zeta \leqslant 1, \zeta\left(x_{1}, y_{1}\right)=1, \zeta(x, y)=0 \quad \text { if }\left|x-x_{1}\right|^{2}+\left|y-y_{1}\right|^{2}>1 \\
|D \zeta| \leqslant 2 \quad \text { in } \mathbf{R}^{n} \times \mathbf{R}^{n}
\end{array}\right.
$$

Finally, set

$$
\Psi(x, y)=\Phi(x, y)+2 \delta \zeta(x, y)=u(x)-v(y)+3 M \beta_{\varepsilon}(x-y)+2 \delta \zeta(x, y) .
$$


We claim that $\Psi$ has a global maximum point $\left(x_{0}, y_{0}\right) \in \mathbf{R}^{n} \times \mathbf{R}^{n}$. Indeed, from (2.11) one deduces

$$
\Psi\left(x_{1}, y_{1}\right)=\Phi\left(x_{1}, y_{1}\right)+2 \delta \geqslant \sup \Phi+\delta
$$

whereas $\lim \sup _{|x|+|y| \rightarrow \infty} \Psi(x, y) \leqslant \sup \Phi$. Moreover, with $\bar{x},\left(x_{0}, y_{0}\right)$ as above,

$$
\begin{aligned}
u\left(x_{0}\right)-v\left(y_{0}\right)+ & 3 M \beta_{\varepsilon}\left(x_{0}-y_{0}\right)+2 \delta \zeta\left(x_{0}, y_{0}\right) \\
& \geqslant u(\bar{x})-v(\bar{x})+3 M+2 \delta \zeta(\bar{x}, \bar{x}),
\end{aligned}
$$

hence, $2 M+3 M \beta_{\varepsilon}\left(x_{0}-y_{0}\right)+2 \delta>3 M$. We conclude that

$$
\left|x_{0}-y_{0}\right| \leqslant \varepsilon \quad \text { if } M>2 \delta \text {. }
$$

Next, using the assumptions on $u, v$ as above, one deduces

$$
u\left(x_{0}\right)+H\left(-3 M D \beta_{\varepsilon}\left(x_{0}-y_{0}\right)-2 \delta D_{x} \zeta\left(x_{0}, y_{0}\right)\right) \leqslant f\left(x_{0}\right)
$$

and

$$
v\left(y_{0}\right)+H\left(-3 M D \beta_{\varepsilon}\left(x_{0}-y_{0}\right)+2 \delta D_{y} \zeta\left(x_{0}, y_{0}\right)\right) \geqslant g\left(y_{0}\right) .
$$

These imply

$$
u\left(x_{0}\right)-v\left(y_{0}\right) \leqslant f\left(x_{0}\right)-g\left(y_{0}\right)+\omega_{H, r}(8 \delta),
$$

where $\omega_{H, r}$ denotes the modulus of continuity of $H$ on $\left\{\xi \in \mathbf{R}^{n} ;|\xi| \leqslant r\right\}$ and $r=3 M\left\|D \beta_{\varepsilon}\right\|+8 \delta$. Therefore, for $x \in \mathbf{R}^{n}$, we deduce

$$
\begin{aligned}
u(x)-v(x)+3 M & \leqslant u(x)-v(x)+3 M+2 \delta \zeta(x, x)=\Psi(x, x) \\
& \leqslant \Psi\left(x_{0}, y_{0}\right) \leqslant u\left(x_{0}\right)-v\left(y_{0}\right)+3 M+2 \delta \\
& \leqslant \sup _{\mathbf{R}^{n}}(f-g)^{+}+\omega_{g}(\varepsilon)+\omega_{H, r}(8 \delta)+2 \delta+3 M .
\end{aligned}
$$

We conclude upon sending $\delta \rightarrow 0$ and then $\varepsilon \rightarrow 0$.

REMARK 2.2. It is worth noting that the essential ingredients of the above proofs are, in fact, general results on the semidifferentials of $u, v \in C_{b}\left(\mathbf{R}^{n}\right)$. Indeed, we actually proved the following in the course of the proof of Theorem 2.1.

Proposition 2.1. Let $u, v \in C\left(\mathbf{R}^{n}\right)$.

(a) If $u$, $v$ satisfy (2.3) and $\sup _{\mathbf{R}^{n}}(u-v)>0$, then for each $\gamma>0$ there exist $x_{0}$, $y_{0} \in \mathbf{R}^{n}$ satisfying

$$
\left\{\begin{array}{l}
u\left(x_{0}\right)-v\left(y_{0}\right) \geqslant \sup _{\mathbf{R}^{n}}(u-v), \quad\left|x_{0}-y_{0}\right| \leqslant \gamma, \quad \text { and } \\
D^{+} u\left(x_{0}\right) \cap D^{-} v\left(y_{0}\right) \quad \text { is not empty. }
\end{array}\right.
$$

(b) Let $u, v \in C_{b}\left(\mathbf{R}^{n}\right)$ and $\sup (u-v)>0$. Let $M=\max (\|u\|,\|v\|)$. Then there is $a$ constant $K>0$, independent of $u$ and $v$, such that for $0<\gamma, \lambda \leqslant 1$ there are $x_{0}$, $y_{0} \in \mathbf{R}^{N}$ satisfying

$$
\left\{\begin{array}{l}
\text { (i) }\left|x_{0}-y_{0}\right| \leqslant \gamma \text { and } u\left(x_{0}\right)-v\left(y_{0}\right) \geqslant \sup (u-v)-\lambda M \\
\text { (ii) there exist } p \in D^{+} u\left(x_{0}\right), q \in D^{-} v\left(y_{0}\right) \\
\text { satisfying }|p|,|q| \leqslant K M(1 / \gamma+\lambda) \text { and }|p-q| \leqslant K \lambda M
\end{array}\right.
$$


Of course, if $u, v \in C^{1}\left(\mathbf{R}^{n}\right)$ in case (a) and if $x_{0}$ is a maximum point of $h=u-v$, then $x_{0}=y_{0}$ solves (2.16) with $\gamma=0$. The $C^{1}$ version of $(2.17)$ is that if $h \in C^{1}(\mathbf{R}) \cap$ $C_{b}(\mathbf{R})$, then one can solve $h\left(x_{0}\right) \geqslant \sup h-\gamma,\left|D h\left(x_{0}\right)\right|<\gamma$ for each $\gamma>0$. The uniqueness result, Theorem 2.1, is an easy consequence of Proposition 2.1. For example, if $u, v$ satisfy (2.3) and are viscosity solutions of $u+H(D u)=f$ and $v+H(D v)=g$ respectively, we then deduce

$$
u\left(x_{0}\right)+H(p) \leqslant f\left(x_{0}\right), \quad v\left(y_{0}\right)+H(p) \geqslant g\left(y_{0}\right)
$$

where $x_{0}, y_{0}$ are as in (2.16) and $p$ is the common element of $D^{+} u\left(x_{0}\right)$ and $D^{-} v\left(y_{0}\right)$. Thus

$$
\sup _{\mathbf{R}^{n}}(u-v) \leqslant f\left(x_{0}\right)-g\left(y_{0}\right) \leqslant \sup _{\mathbf{R}^{n}}(f-g)+\omega_{g}(\gamma)
$$

and we conclude by letting $\gamma \rightarrow 0$.

The point of these remarks is that proofs of facts about viscosity solutions can, in fact, be regarded as applications of general results concerning the generalized calculus of the semidifferentials $D^{+}, D^{-}$to problems at hand.

3. Existence of viscosity solutions. To demonstrate the existence of viscosity solutions (and also explain the name of these solutions) we quickly reprove the following

THEOREM 3.1. Let $\varepsilon>0$, and let $F_{\varepsilon}(y, t, p)$ be a family of continuous functions such that $F_{\varepsilon}(y, t, p)$ converges uniformly on compact subsets of $\theta \times \mathbf{R} \times \mathbf{R}^{n}$ to some function $F(y, t, p)$, as $\varepsilon$ goes to 0 . Finally, suppose $u^{\varepsilon} \in C^{2}(\Theta)$ is a solution of

$$
-\varepsilon \Delta u^{\varepsilon}+F_{\varepsilon}\left(y, u^{\varepsilon}, D u^{\varepsilon}\right)=0 \text { in } \Theta,
$$

and let us assume that the $u^{\varepsilon}$ converge uniformly on compact subsets of $\mathcal{E}$ to some $u \in C(\theta)$. Then $u$ is a viscosity solution of $(0.1)$.

Remark 3.1. This result is proved in [2, Proposition IV.1]. (See also [5, pp. 237-238].)

Proof of Theorem 3.1. Let us check (0.7) first for $\phi \in C^{2}(\theta)$. Assuming that $u-\phi$ has a local maximum at $y_{0} \in \theta$, choose $\zeta \in C^{\infty}(\theta)$ such that $0 \leqslant \zeta<1$ if $y \neq y_{0}, \zeta\left(y_{0}\right)=1$. Obviously, $u-(\phi-\zeta)$ has a strict local maximum at $y_{0} \in \Theta$ and thus for $\varepsilon$ small enough, $u^{\varepsilon}-(\phi-\zeta)$ has a local maximum at some $y_{\varepsilon} \in \mathcal{E}$ and $y_{\varepsilon} \rightarrow y_{0}$ as $\varepsilon \rightarrow 0$. But at the point $y=y_{\varepsilon}$, we have

$$
D u^{\varepsilon}\left(y_{\varepsilon}\right)=D(\phi-\zeta)\left(y_{\varepsilon}\right), \quad \Delta u^{\varepsilon}\left(y_{\varepsilon}\right) \leqslant \Delta(\phi-\zeta)\left(y_{\varepsilon}\right)
$$

therefore

$$
F_{\varepsilon}\left(y_{\varepsilon}, u^{\varepsilon}\left(y_{\varepsilon}\right), D(\phi-\zeta)\left(y_{\varepsilon}\right)\right) \leqslant \varepsilon \Delta(\phi-\zeta)\left(y_{\varepsilon}\right) .
$$

We may conclude since $u^{\varepsilon}\left(y_{\varepsilon}\right) \rightarrow u\left(y_{0}\right), D(\phi-\zeta)\left(y_{\varepsilon}\right) \rightarrow D(\phi-\zeta)\left(y_{0}\right)=D \phi\left(y_{0}\right)$, $\varepsilon \Delta(\phi-\zeta)\left(y_{\varepsilon}\right) \rightarrow 0$. Next, if $\phi \in C^{1}(\theta)$ and if $(u-\phi)$ has a local maximum at $y_{0} \in \mathcal{\Theta}$, we again prove $(0.7)$. Take $\phi_{n} \in C^{2}(\theta)$ such that $\phi_{n} \rightarrow \phi$ in $C^{1}(\theta)$ and, as before, choose $\zeta \in C^{\infty}(0)$ such that $0 \leqslant \zeta<1$ if $y \neq y_{0}, \zeta\left(y_{0}\right)=1$. For $n$ large enough, $u-\left(\phi_{n}-\zeta\right)$ has a local maximum at some point $y_{n} \in \mathcal{\theta}$ and $y_{n} \rightarrow y_{0}$. By the argument made above, we know

$$
F\left(y_{n}, u\left(y_{n}\right), D \phi_{n}\left(y_{n}\right)-D \zeta\left(y_{n}\right)\right) \leqslant 0 .
$$


We finish by noting $D \phi_{n}\left(y_{n}\right)-D \zeta\left(y_{n}\right) \rightarrow D \phi\left(y_{0}\right)-D \zeta\left(y_{0}\right)=D \phi\left(y_{0}\right)$.

As an illustration of the above result, we prove

Corollary 3.1. Assume $H: \mathbf{R}^{n} \rightarrow \mathbf{R}$ is continuous, $\lambda>0$. Then for each $f \in$ $\operatorname{BUC}\left(\mathbf{R}^{n}\right)=X,{ }^{4}$ there exists a unique viscosity solution of $u \in X$ of $u+\lambda H(D u)=$ $f(x)$ in $\mathbf{R}^{n}$. Let us denote this solution by $u=R_{\lambda} f$. Then for all $f, g \in \operatorname{BUC}\left(\mathbf{R}^{n}\right)$, we have

$$
\left\|\left(R_{\lambda} f-R_{\lambda} g\right)^{+}\right\| \leqslant\left\|(f-g)^{+}\right\| .
$$

Proof. The uniqueness and the estimate (3.1) are immediate consequences of Theorem 2.1. To prove the existence, we consider the approximate problem

$$
u^{\varepsilon}+\lambda H\left(D u^{\varepsilon}\right)-\varepsilon \Delta u^{\varepsilon}=f^{\varepsilon} \quad \text { in } \mathbf{R}^{n}
$$

where $H_{\varepsilon}, f^{\varepsilon} \in C^{\infty}\left(\mathbf{R}^{n}\right)$ and $H_{\varepsilon} \rightarrow H$ in $C\left(\mathbf{R}^{n}\right)$ as $\varepsilon \rightarrow 0, f^{\varepsilon} \rightarrow f$ in $C_{b}\left(\mathbf{R}^{N}\right)$ as $\varepsilon \rightarrow 0$. Routine P.D.E. arguments imply the existence of a unique smooth solution $u^{\varepsilon} \in$ $\operatorname{BUC}\left(\mathbf{R}^{n}\right)$ (see, for example, $\left.[7,3,8]\right)$. In addition if, $v^{\varepsilon} \in X$ solves $v^{\varepsilon}+\lambda H\left(D v^{\varepsilon}\right)-$ $\varepsilon \Delta v^{\varepsilon}=g^{\varepsilon}$ in $\mathbf{R}^{n}$, the maximum principle yields

$$
\left\|u^{\varepsilon}-v^{\varepsilon}\right\| \leqslant\left\|f^{\varepsilon}-g^{\varepsilon}\right\| .
$$

If we take $g^{\varepsilon}(X)=f^{\varepsilon}(x+y)$ for some fixed $y \in \mathbf{R}^{n}$, we obtain

$$
\sup _{\mathbf{R}^{n}}\left|u^{\varepsilon}(x)-u^{\varepsilon}(x+y)\right| \leqslant\left\|f^{\varepsilon}-g^{\varepsilon}\right\| ;
$$

that is,

$$
\omega_{u} \varepsilon(\cdot) \leqslant \omega_{f} \varepsilon(\cdot) .
$$

Thus the set $\left\{u^{\varepsilon}\right\}$ is bounded in $C_{b}\left(\mathbf{R}^{n}\right)$ and equicontinuous, and there exists $\varepsilon_{j} \rightarrow 0$ and $u \in X$ such that $u^{\varepsilon_{j}} \rightarrow_{j} u$ locally uniformly. Now recall Theorem 3.1.

REMARK 3.1 For more general existence results, we refer to P. L. Lions [8].

As a final result in this section, we obtain an estimate on a bounded viscosity solution $u$ of

$$
u+H(D u) \leqslant f
$$

under the assumptions

$$
|H(p)| \leqslant L|p|, \quad p \in \mathbf{R}^{n}
$$

and

$$
|f(x)| \leqslant a e^{-b|x|}, \quad x \in \mathbf{R}^{n},
$$

where $L, a, b$ are nonnegative constants. Set

$$
v(x)=c e^{-d|x|}
$$

where $c, d>0$. Clearly

$$
D^{-} v(x)= \begin{cases}-c d e^{-d|x|} x /|x| & \text { if } x \neq 0 \\ \text { empty } & \text { if } x=0\end{cases}
$$

${ }^{4} \mathrm{BUC}\left(\mathbf{R}^{n}\right)=\left\{u \in C_{b}\left(\mathbf{R}^{n}\right), u\right.$ is uniformly continuous on $\left.\mathbf{R}^{n}\right\}$ 
Thus $v$ is a viscosity supersolution of $v+H(D v)=f$ provided that

$$
c e^{-d|x|}+H\left(-c d e^{-d|x|} x /|x|\right) \geqslant f(x) \text { for } x \neq 0 .
$$

In view of (3.6) and (3.7), the estimate (3.10) is valid provided $c e^{-d|x|}-L c d e^{-d|x|} \geqslant$ $a e^{-b|x|}$ or, equivalently $c(1-L d) \geqslant a e^{(d-b)|x|}$. If $d \leqslant b$ and $L d<1$ we conclude that $v=a /(1-L d) e^{-d|x|}$ is a supersolution of $v+H(D v)=f$. The proof of (2.2) of Theorem 2.1 used only that $u$ is a subsolution and $v$ is a supersolution; so we conclude that (3.5), (3.6), (3.7) imply

$$
u(x) \leqslant \frac{a}{1-L d} e^{-d|x|} \text { for } d<b, L d<1 .
$$

Compare this with the proof of [6, Lemma 2.2].

4. Uniqueness for $u_{t}+H(D u)=0$. This section and the next one concern the Hamilton-Jacobi equation

$$
u_{t}+H(D u)=0 \quad \text { in } \mathbf{R}^{n} \times(0, \infty),
$$

with the initial condition

$$
u(x, 0)=u_{0}(x), \quad x \in \mathbf{R}^{n} .
$$

According to our definitions and to Theorem $1.1, u \in C\left(\mathbf{R}^{n} \times(0, T)\right)$ is a viscosity solution of

$$
u_{t}+H(D u)=0 \quad \text { on } \mathbf{R}^{n} \times(0, T)
$$

provided that, for every $\phi \in C^{1}\left(\mathbf{R}^{n} \times(0, T)\right)$, we have $\phi_{t}+H(D \phi) \leqslant 0$ (resp. $\geqslant 0$ ) at local maxima (resp. minima) of $u-\phi$. We will prove

TheOREM 4.1. Let $0<T<\infty$ and let $u, v \in \mathrm{BUC}\left(\mathbf{R}^{n} \times[0, T]\right)$ be viscosity solutions of (4.3). Then

$$
\sup _{\mathbf{R}^{n} \times[0, T]}(u-v)^{+} \leqslant \sup _{\mathbf{R}^{n}}(u(x, 0)-v(x, 0))^{+} .
$$

REMARK 4.1. The method of $\S 3$ is easily adapted to prove that if $u_{0} \in \operatorname{BUC}\left(\mathbf{R}^{n}\right)$, then (4.1), (4.2) has a unique viscosity solution $u$ such that $u \in \operatorname{BUC}\left(\mathbf{R}^{n} \times[0, T]\right)$ for every $0<T<\infty$. This will also follow from $\S 5$ and nonlinear semigroup theory.

Proof of Theorem 4.1. We will give the proof in the general case. However, let us first dispose of the following technicality.

Lemma 4.1. Let $0<T<\infty$ and let $u \in C\left(\mathbf{R}^{n} \times(0, T)\right)$ be a viscosity solution of (4.3). If $\phi \in C^{1}\left(\mathbf{R}^{n} \times(0, T]\right)$, then at each local maximum point (resp. minimum point ) of $u-\phi$ on $\mathbf{R}^{n} \times(0, T]$, we have $\phi_{t}+H(D \phi) \leqslant 0$ (resp. $\left.\geqslant 0\right)$.

Proof. The point is that assumptions on $(0, T)$ imply conclusions on $(0, T]$; this is because of the special dependence of the equation on the time derivative. For example, we prove that if $\left(x_{0}, T\right)$ is a local maximum in $\mathbf{R}^{n} \times(0, T]$ of $u-\phi$ with $\phi \in C^{1}\left(\mathbf{R}^{n} \times(0, T]\right)$, then $\phi_{t}\left(x_{0}, T\right)+H\left(D \phi\left(x_{0}, T\right)\right) \leqslant 0$. As observed many times above, we may assume $\left(x_{0}, T\right)$ is a strict local maximum of $u-\phi$ on $\mathbf{R}^{n} \times(0, T]$. Then we choose $\mu, r>0,0<r<T$, so that

$$
u(x, t)-\phi(x, t) \leqslant u\left(x_{0}, T\right)-\phi\left(x_{0}, T\right)-\mu
$$


for

$$
\begin{aligned}
(x, t) \in K_{r}=\{(x, t): & \left|x-x_{0}\right|=r \text { and } \\
& \left.0 \leqslant T-t \leqslant r \text { or }\left|x-x_{0}\right| \leqslant r \text { and } t=T-r\right\} .
\end{aligned}
$$

If $\varepsilon>0$ is small, it is then obvious that $\psi_{\varepsilon}(x, t)=u(x, t)-\phi(x, t)-\varepsilon(T-t)^{-1}$ has a local maximum point $\left(x_{\varepsilon}, t_{\varepsilon}\right)$ such that $t_{\varepsilon}<T$ and $\left(x_{\varepsilon}, t_{\varepsilon}\right) \rightarrow\left(x_{0}, T\right)$ as $\varepsilon \rightarrow 0$. By assumption we then have

$$
\phi_{t}\left(x_{\varepsilon}, t_{\varepsilon}\right)+\frac{\varepsilon}{\left(T-t_{\varepsilon}\right)^{2}}+H\left(D \phi\left(x_{\varepsilon}, t_{\varepsilon}\right)\right) \leqslant 0 .
$$

This implies $\phi_{t}\left(x_{\varepsilon}, t_{\varepsilon}\right)+H\left(D \phi\left(x_{\varepsilon}, t_{\varepsilon}\right)\right) \leqslant 0$ and the result follows upon letting $\varepsilon \rightarrow 0$.

We may now begin the proof of Theorem 4.1, which involves the construction of a rather complicated "test function". Define $\sigma$ by

$$
\sup _{\mathbf{R}^{n} \times[0, T]}(u-v)=\sup _{\mathbf{R}^{n}}\left(u_{0}(x)-v_{0}(x)\right)+\sigma,
$$

where here and below $u_{0}=u(\cdot, 0), v_{0}=v(\cdot, 0)$. If $\sigma=0$, there is nothing to prove, and so we may assume $\sigma>0$.

Choose $\beta \in C^{\infty}\left(\mathbf{R}^{n} \times \mathbf{R}\right)$ so that

$$
\left\{\begin{array}{l}
0 \leqslant \beta \leqslant 1, \quad \beta(0,0)=1 \quad \text { and } \\
\beta(x, t)=0 \quad \text { if }|x|^{2}+t^{2}>1 .
\end{array}\right.
$$

Set $\beta_{\varepsilon}(x, t)=\beta(x / \varepsilon, t / \varepsilon)$ and $M=\max (\|u\|,\|v\|)$, where $\|h\|$ denotes the norm of $h$ in $C_{b}\left(\mathbf{R}^{n} \times[0, T]\right)$ (note that since $\sigma>0, M>0$ ). Next, let $\lambda>0$ be fixed and define $\Phi: \mathbf{R}^{n} \times \mathbf{R}^{n} \times[0, T] \times[0, T] \rightarrow \mathbf{R}$ by

$$
\Phi(x, y, t, s)=u(x, t)-v(y, s)-\lambda(t+s)+(5 M+2 \lambda T) \beta_{\varepsilon}(x-y, t-s) .
$$

If $\Phi$ attains its maximum on $\mathbf{R}^{2 n} \times[0, T]^{2}$ at some point, the proof is easily completed, but this need not be so. Therefore we choose $\delta>0$ and then $\left(x_{0}, y_{0}, t_{0}, s_{0}\right) \in \mathbf{R}^{2 n} \times[0, T]^{2}$ so that

$$
\Phi\left(x_{0}, y_{0}, t_{0}, s_{0}\right)>\sup \Phi-\delta .
$$

The first claim is that if $\lambda, \varepsilon$ and $\delta$ are sufficiently small, then for some $\mu>0$,

$$
t_{0}, s_{0} \geqslant \mu,
$$

where $\mu$ is independent of $\lambda, \varepsilon, \delta$. To see this, first note

$$
\left\{\begin{array}{l}
\Phi(x, y, t, s) \leqslant 2 M \quad \text { if }|x-y|^{2}+|t-s|^{2} \geqslant \varepsilon^{2}, \\
\sup _{\mathbf{R}^{2 n} \times[0, T]^{2}} \Phi \geqslant \sup _{\mathbf{R}^{n}} \Phi(x, x, T, T) \geqslant 3 M .
\end{array}\right.
$$


Hence, if $\delta<M$, then $\left|x_{0}-y_{0}\right|^{2}+\left(t_{0}-s_{0}\right)^{2}<\varepsilon^{2}$. Therefore for $\delta<M$,

$$
\begin{aligned}
\Phi\left(x_{0}, y_{0}, t_{0}, s_{0}\right)= & u\left(x_{0}, t_{0}\right)-v\left(y_{0}, s_{0}\right)-\lambda\left(t_{0}+s_{0}\right) \\
& +(5 M+2 \lambda T) \beta_{\varepsilon}\left(x_{0}-y_{0}, t_{0}-s_{0}\right) \\
\leqslant & u\left(x_{0}, t_{0}\right)-v\left(x_{0}, t_{0}\right)-\lambda\left(t_{0}+s_{0}\right)+5 M+2 \lambda T+\omega_{v}(\varepsilon) \\
\leqslant & u\left(x_{0}, 0\right)-v\left(x_{0}, 0\right)-\lambda\left(t_{0}+s_{0}\right)+5 M+2 \lambda T \\
& +\omega_{u}\left(t_{0}\right)+\omega_{v}\left(t_{0}\right)+\omega_{v}(\varepsilon) .
\end{aligned}
$$

Since on the other hand we have, in view of (4.6),

$$
\sup \Phi(x, x, t, t) \geqslant \sup (u(x, t)-v(x, t))+5 M=\sup \left(u_{0}-v_{0}\right)+\sigma+5 M,
$$

we finally obtain

$$
2 \lambda T+\omega_{u}\left(t_{0}\right)+\omega_{v}\left(t_{0}\right)+\omega_{v}(\varepsilon) \geqslant \sigma-\delta .
$$

Now if $\delta<\sigma / 4, \varepsilon$ is small enough to force $\omega_{v}(\varepsilon)<\sigma / 4,2 \lambda T<\sigma / 4$, and $\mu$ is chosen so that $\omega_{u}(\xi)+\omega_{v}(\xi) \leqslant \sigma / 4$ for $0<\xi \leqslant \mu$, we conclude $t_{0} \geqslant \mu$. Similarly we obtain $s_{0} \geqslant \mu$ and (4.10) is proved.

Next select $\zeta \in C^{\infty}\left(\mathbf{R}^{2 n} \times[0, T]^{2}\right)$ satisfying $0 \leqslant \zeta \leqslant 1, \zeta\left(x_{0}, y_{0}, t_{0}, s_{0}\right)=1, \zeta=$ 0 if $\left|x-x_{0}\right|^{2}+\left|y-y_{0}\right|^{2}+\left|t-t_{0}\right|^{2}+\left|s-s_{0}\right|^{2} \geqslant \mu^{2} / 4$. We set $\Psi(x, y, t, s)=$ $\Phi(x, y, t, s)+2 \delta \zeta(x, y, t, s)$. Since $\Psi \leqslant \Phi$ off the support of $\zeta$ and since

$$
\Psi\left(x_{0}, y_{0}, t_{0}, s_{0}\right)=\Phi\left(x_{0}, y_{0}, t_{0}, s_{0}\right)+2 \delta>\sup \Phi+\delta,
$$

$\Psi$ attains its maximum at some point $\left(x_{1}, y_{1}, t_{1}, s_{1}\right)$, which lies in the support of $\zeta$. Thus $t_{1}, s_{1} \geqslant \mu / 2$. But $\left(x_{1}, t_{1}\right) \in \mathbf{R}^{n} \times[0, T]$ is a maximum of $(x, t) \rightarrow u(x, t)-$ $v\left(y_{1}, s_{1}\right)-\lambda\left(t+s_{1}\right)+(5 M+2 \lambda T) \beta_{\varepsilon}\left(x-y_{1}, t-s_{1}\right)+2 \delta \zeta\left(x, y_{1}, t, s_{1}\right)$, so that Lemma 4.1 implies

$$
\begin{aligned}
\lambda-(5 M+ & 2 \lambda T) \frac{\partial \beta_{\varepsilon}}{\partial t}\left(x_{1}-y_{1}, t_{1}-s_{1}\right)-2 \delta \zeta_{t}\left(x_{1}, y_{1}, t_{1}, s_{1}\right) \\
& +H\left(-(5 M+2 \lambda T) D_{x} \beta_{\varepsilon}\left(x_{1}-y_{1}, t_{1}-s_{1}\right)-2 \delta D_{x} \zeta\left(x_{1}, y_{1}, t_{1}, s_{1}\right)\right) \leqslant 0 .
\end{aligned}
$$

Similarly,

$$
\begin{aligned}
-\lambda+(5 M & +2 \lambda T) \frac{\partial \beta_{\varepsilon}}{\partial t}\left(x_{1}-y_{1}, t_{1}-s_{1}\right)+2 \delta \zeta_{s}\left(x_{1}, y_{1}, t_{1}, s_{1}\right) \\
& +H\left(-(5 M+2 \lambda T) D_{x} \beta_{\varepsilon}\left(x_{1}-y_{1}, t_{1}-s_{1}\right)+2 \delta D_{y} \zeta\left(x_{1}, y_{1}, t_{1}, s_{1}\right)\right) \geqslant 0 .
\end{aligned}
$$

Combining these two inequalities and letting $\delta \rightarrow 0$, we derive $\lambda=0$, a contradiction.

REMARK 4.2. The assumption $u, v \in \mathrm{BUC}\left(\mathbf{R}^{n} \times[0, T]\right)$ was used in the proof, but minor modifications allow one to weaken this to $u_{0}, v_{0} \in \mathrm{BUC}\left(\mathbf{R}^{n}\right), u(x, t) \rightarrow u_{0}(x)$, $v(x, t) \rightarrow v_{0}(x)$ uniformly on $\mathbf{R}^{n}$ as $t \rightarrow 0$.

REMARK 4.3. Theorem 4.1 is a special case of [2, Theorem V.2]. The proof in [2] is, however, only indicated and involves a more cumbersome comparison. See [2, § V.3] concerning domains of dependence.

5. The semigroup approach. Let $H \in C\left(\mathbf{R}^{n}\right)$, and $X=\mathrm{BUC}\left(\mathbf{R}^{n}\right)$. We now realize the formal expression " $H(D u)$ " as a nonlinear operator on $X$. 
Definition. We say that $u \in X$ belongs to $D(A)$ (the domain of $A$ ) if $u$ is a viscosity solution of $H(D u)=f$ in $\mathbf{R}^{n}$; for some $f \in X$. We denote by $A u$ the set of all such $f \in X$.

Obviously, $D(A) \supset\{u \in X, D u \in X\}$ (because of the consistency result Theorem 1.2) and so $\overline{D(A)}=X$.

REMARK 5.1. Except in very special cases we do not know whether $A$ is single valued. (See L. C. Evans [6] when $H$ is uniformly continuous on $\mathbf{R}^{n}$; the case when $H(p) \rightarrow \infty$ as $|p| \rightarrow \infty$ is easily deduced from the results of M. G. Crandall and P. L. Lions [2].) For simplicity, we write as if $A$ were single valued below.

Proposition 5.1. A is an m-accretive operator on $X$.

Remark 5.2. See Barbu [1] or L. C. Evans [5, Appendix 1] for definitions.

Proof of Proposition 5.1. In view of Corollary 3.1 we have $R(I+\lambda A)=X$ (for $\lambda>0)$ and by (3.1), $\|u-v\| \leqslant\|u-v+\lambda(A u-A v)\|$ if $\lambda>0, u, v \in D(A)$.

Proposition 5.1 implies, by the Crandall-Liggett generation theorem (cf. [1]), that $A$ generates a nonlinear semigroup of contractions $(S(t))_{t \geqslant 0}$ on $X$ and

$$
S(t) u_{0}=\lim _{\substack{n \rightarrow \infty \\ n \lambda \rightarrow t}}(I+\lambda A)^{-n} u_{0}, \quad u_{0} \in X=\overline{D(A)},
$$

uniformly for $t$ in bounded subsets of $(0, \infty)$. Furthermore, the mapping $t \mapsto S(t) u_{0}$ is continuous from $[0, \infty)$ into $X$.

In general the semigroup generated by a (nonlinear) $m$-accretive operator $A$ can be regarded as a "mild" solution of the evolution equation

$$
\frac{d u}{d t}+A u=0 \quad(t>0), \quad u(0)=u_{0}
$$

see the forthcoming book of P. Benilan, M. G. Crandall and A. Pazy [1]. For the case at hand a stronger interpretation is possible.

Proposition 5.2. Assume $u_{0} \in X$. Then

$$
u(x, t)=\left(S(t) u_{0}\right)(x), \quad 0 \leqslant t \leqslant T, x \in \mathbf{R}^{n},
$$

is the viscosity solution of (4.1), (4.2).

Proof. Assume $\phi \in C^{1}\left(\mathbf{R}^{n} \times(0, T)\right)$ and $u-\phi$ attains a local maximum at $\left(x_{0}, t_{0}\right) \in \mathbf{R}^{n} \times(0, T)$. We may as well assume $\left(x_{0}, t_{0}\right)$ is a strict local maximum of $u-\phi$ in view of arguments used above.

For each $\varepsilon>0$, consider the step function $u^{\varepsilon}(t)$ solving

$$
\left\{\begin{array}{l}
\frac{1}{\varepsilon}\left\{u^{\varepsilon}(t+\varepsilon)-u^{\varepsilon}(t)\right\}+A u^{\varepsilon}(t+\varepsilon)=0, \quad t \geqslant 0, \\
u^{\varepsilon}(t)=u_{0} \text { if } 0 \leqslant t \leqslant \varepsilon .
\end{array}\right.
$$

We may assume $t_{0} \neq k \varepsilon$ for any integer $k$ by appropriate choice of $\varepsilon$. Since $u^{\varepsilon}(t) \rightarrow S(t) u_{0}$ uniformly on $[0, T]$ in $X$ as $\varepsilon \rightarrow 0, u^{\varepsilon}(x, t+\varepsilon)-\phi(x, t)$ has a local maximum at some point $\left(x^{\varepsilon}, t^{\varepsilon}\right)$, such that $\left(x^{\varepsilon}, t^{\varepsilon}\right) \in \mathbf{R}^{n} \times(0, T), x^{\varepsilon} \rightarrow x_{0}, t^{\varepsilon} \rightarrow t_{0}$ as $\varepsilon \rightarrow 0$. Hence,

$$
A u^{\varepsilon}\left(t^{\varepsilon}+\varepsilon\right)-A \phi \geqslant 0 \quad \text { at } x_{\varepsilon}
$$


according to the definition of $A$ (note $u^{\varepsilon}(d o t+\varepsilon) \in D(A)$ ). Also, if $\varepsilon$ is small enough, we have

$$
\frac{1}{\varepsilon}\left(u^{\varepsilon}\left(x^{\varepsilon}, t^{\varepsilon}+\varepsilon\right)-u^{\varepsilon}\left(x^{\varepsilon}, t^{\varepsilon}\right)\right) \geqslant \frac{1}{\varepsilon}\left(\phi\left(x^{\varepsilon}, t^{\varepsilon}\right)-\phi\left(x^{\varepsilon}, t^{\varepsilon}-\varepsilon\right)\right) .
$$

Combining this inequality with (5.3) we deduce

$$
A \phi\left(x^{\varepsilon}, t^{\varepsilon}\right)=H\left(D \phi\left(x^{\varepsilon}, t^{\varepsilon}\right)\right) \leqslant \frac{1}{\varepsilon}\left(\phi\left(x^{\varepsilon}, t^{\varepsilon}-\varepsilon\right)-\phi\left(x^{\varepsilon}, t^{\varepsilon}\right)\right) .
$$

If we let $\varepsilon \rightarrow 0$, then, since $\left(x^{\varepsilon}, t^{\varepsilon}\right) \rightarrow\left(x_{0}, t_{0}\right)$, we finally obtain

$$
\phi_{t}\left(x_{0}, t_{0}\right)+H\left(D \phi\left(x_{0}, t_{0}\right)\right) \leqslant 0 .
$$

The opposite inequality has an analogous proof should $u-\phi$ attain a local minimum at $\left(x_{1}, t_{1}\right)$.

This result corresponds to [2, §VI. 3].

\section{REFERENCES}

1. V. Barbu, Nonlinear semigroups and differential equations in Banach spaces, Noordhoff, Leyden, 1976.

2. M. G. Crandall and P. L. Lions, Viscosity solutions of Hamilton-Jacobi equations, Trans. Amer. Math. Soc. 277 (1983), 1-42.

3. Condition d'unicité pour les solutions généralisées des équations de Hamilton-Jacobi du premier ordre, C. R. Acad. Sci. Paris Sér. A-B 292 (1981), 183-186.

4. E. DeGiorgi, A. Marino and M. Tosques, Problemi di evoluzione in spazi metrici e curve di massima pendenza, Rend. Classe Sci. Fis. Mat. Nat. Accad. Naz. Lincei 68 (1980), 180-187.

5. L. C. Evans, On solving certain nonlinear partial differential equations by accretive operator methods, Israel J. Math. 36 (1981), 235-247.

6. Some max-min methods for the Hamilton-Jacobi equation, Indiana Univ. Math. J. (to appear).

7. A. Friedman, The Cauchy problem for first order partial differential equations, Indiana Univ. Math. J. 23 (1973), 27-40.

8. P. L. Lions, Generalized solutions of Hamilton-Jacobi equations, Pitman, London, 1982.

Mathematics Research Center, University of Wisconsin, Madison, Wisconsin 53706

Department of Mathematics, University of Maryland, College Park, Maryland 20742

Ceremade Universite Paris-IX, Dauphine, Place De-Lattre-de-Tassigny, 75775 Paris, France 